\title{
Novel multiplex-PCR for rapid detection of Bacillus anthracis spores present in soils of Sirajganj district in Bangladesh
}

\author{
KHMNH Nazir $^{1 *}$, Hassan $^{1}$, SMZH Chowdhury ${ }^{2}$, MB Rahman ${ }^{1}$ \\ ${ }^{1}$ Department of Microbiology and Hygiene, Bangladesh Agricultural University, Mymensingh-2202, \\ Bangladesh; ${ }^{2}$ Bangladesh Agricultural Research Council, Farmgate, Dhaka, Bangladesh
}

\begin{abstract}
Bacillus anthracis spores were isolated and detected from soil samples $(\mathrm{n}=72)$ using multiplex-PCR method. The bacteria were isolated and primarily identified as Bacillus anthracis using selective Polymyxin B Lysozyme - EDTA - Thallous acetate agar. A multiplex-PCR method targeting three genes; rpoB of genome, pag of pX01 and cap of pX02 was done to confirm the isolated bacteria. Among 72 soil samples, the viable $B$. anthracis spores could be extracted from 14 (19.44\%) samples. However, both pX01 and pX02 plasmids were harbored in 5 (6.94\%) isolates. On the other hand, pX01 and pX02 was present in 8 (57.14\%) and 11 (78.57\%) isolates, respectively. This two-step-method was found to be easy, accurate and rapid in identification of $B$. anthracis spores from soil samples and to identify the toxigenic plasmids present in this bacterium.
\end{abstract}

Key words: Anthrax, Bacillus anthracis, spore, multiplex-PCR, soil

Progressive Agriculturists. All rights reserve

*Corresponding Author: nazir@bau.edu.bd

\section{Introduction}

Bacillus anthracis, the causal agent of anthrax, is a soil-borne, spore forming bacterium. The bacterium forms a dormant stage called endospore when it comes in contact with unfavorable atmosphere (Dragon and Rennie, 2001). The spores may persist in the environment for several decades and the susceptible animal may be infected after ingestion of the spores through contaminated feed or water (Fasanella et al., 2013). Virulent strain of the bacterium possesses toxin-encoding plasmids; pX01 (181.7 kb) and/or pX02 (96.2 kb) (Vahedi et al., 2009). The pX01 contains three different genes namely protective antigen (pag), lethal factor (lef), oedema factor (cya), whereas pX02 contains capsule (cap) gene (Park et al., 2007; Kumar et al., 2012).

Anthrax is found as a common problem of animal and subsequently to human in tropical and subtropical countries including Bangladesh (Biswas et al., 2011). In Bangladesh, the disease is considered as an enzootic and the outbreak is mostly occurred in
Sirajganj, a north-western district (Ahmed et al., 2010; Biswas et al., 2011; Fasanella et al., 2013; Ahsan et al., 2013). Previously, investigation of anthrax in Bangladesh was limited within surveillance studies during active epizootics (Ahmed et al., 2010; Biswas et al., 2011). Recently, we investigated different environmental parameters (e.g., organic matter, $\mathrm{pH}$, Ca contents of the soil, temperature, and rainfall) and found that some of them are significantly correlated with the repeated anthrax outbreak in Sirajganj, Bangladesh (Ahsan et al., 2013; Hassan et al., 2015a). However, identification of the bacteria was done by a series of complicated and time-consuming traditional bacteriological and biochemical methods. Considering the public health importance, very accurate and quick identification of pathogenic $B$. anthracis is needed. Multiplex-PCR has been used as a useful method for the identification and differentiation of pathogenic B. anthracis from other 
related pathogenic/nonpathogenic bacilli (Ko et al., 2003; Park et al., 2007; Kumar et al., 2012). In this study, taking an advantage of multiplex-PCR method, we established a two-step-method for an easy, precise and rapid identification of $B$. anthracis spore from soil samples targeting the presence of virulent plasmids (pX01 and/or pX02).

\section{Materials and Methods}

To extract the spores, approximately 400-gm of surface soil samples $(n=72)$ were collected from Sirajganj district, Bangladesh (Ahsan et al., 2013). Using the selective Polymyxin B - Lysozyme EDTA - Thallous acetate (PLET) agar (SigmaAldrich, Switzerland), the viable spores were extracted (Dragon and Rennie, 2001; Ahsan et al., 2013). From the B. anthracis positive plates, colonies (one colony from each) were taken and cultured overnight at $37^{\circ} \mathrm{C}$ in liquid culture media. Total DNA was extracted following the procedures of Jula et al. (2007) with some modifications. Combination of three primers targeting portions of rpoB gene (359bp, specific for Bacillus genus; 208-bp, specific for Bacillus anthracis) present in $B$. anthracis chromosome were used in the multiplex-PCR (Ko et al., 2003). In addition, two pairs of primer sets were designed targeting portions of pag gene of pX01 (287-bp) and cap gene of pX02 (164-bp) by analyzing the nucleotide sequences of these two plasmids collected from GenBank database. The oligonucleotide primers (Table 1) were purchased from the Cosmo Genetech Co., Ltd., South Korea. The multiplex-PCR was performed according to the procedure described by Ko et al. (2003) with some modifications. In brief, the PCR assay was performed in a total reaction volume of $25 \mu \mathrm{l}$ consisting of ca. 50 ng template DNA, $2.5 \mu \mathrm{l}$ of $10 \mathrm{x}$ PCR buffer, 0.2 mM dNTPs mix (SolGent Co., Ltd.), 20 pmole of each primer; and $1.5 \mathrm{U}$ of SolGent ${ }^{\mathrm{TM}}$ Taq DNA Polymerase (SolGent Co., Ltd.). Thirty five cycles of amplification were performed in Thermal Cycler (AB Applied Biosystem) following initial denaturation at $95^{\circ} \mathrm{C}$ for $2 \mathrm{~min}$. Each cycle consisted of a denaturation step at $95^{\circ} \mathrm{C}$ for $30 \mathrm{~s}$, an annealing step at $45^{\circ} \mathrm{C}$ for $30 \mathrm{~s}$, and an extension step at $72^{\circ} \mathrm{C}$ for $30 \mathrm{~s}$, with a final extension at $72^{\circ} \mathrm{C}$ for $1 \mathrm{~min}$.
The PCR products were analyzed by electrophoresis on $2 \%(\mathrm{wt} / \mathrm{vol})$ agarose gels stained with ethidium bromide $(0.5 \mathrm{mg} / \mathrm{ml})$. A previously isolated $B$. subtilis isolate (unpublished) was used as a negative control. The purified PCR fragments generated by using specific primers targeting pX01 and pX02 (Table 1) were validated by sequencing using automatic DNA sequencer (ABI 3730XL; Applied Biosystem). The PCR reaction and condition were same as described above.

\section{Results and Discussion}

Among the 72 soil samples, viable $B$. anthracis endospores could be extracted from 14 (19.44\%) samples. These findings are in support of our previous findings (Ahsan et al., 2013). However, Jule et al. (2007) found only $9.1 \%$ soil samples were contaminated with B. anthracis spore in Iran. This variation may be due to difference in geographical location, soil composition, soil $\mathrm{pH}$, humidity, rainfall etc. (Ahsan et al., 2013). On PLET agar medium, the bacteria form circular, creamy-white colonies with ground glass texture. The PLET medium is a selective medium for $B$. anthracis that inhibits all other contaminants including B. cereus (Dragon and Rennie, 2001). However, in our previous study, the bacteria were identified through a series of timeconsuming and laborious steps (Jula et al., 2007; Vahedi et al., 2009; Ahsan et al., 2013; Hassan et al., 2015b). Identification of $B$. anthracis by PCR targeting only toxin-encoding genes (pX01 or pX02) may not give accurate results in identification of $B$. anthracis as the plasmid can be transferred among closest species by horizontal gene transfer (Ko et al., 2003). Moreover, all the B. anthracis isolates may not contain these toxin-encoding genes and in some cases the plasmids can be lost naturally (Ko et al., 2003; Vahedi et al., 2009). Therefore, combination of B. anthracis specific chromosomal marker along with toxin-encoding gene amplification would be an authentic and reliable strategy for the specific detection of this species. Here, an easy, precise and rapid identification of $B$. anthracis spore from soil samples has been established that enabled us to avoid hazards of handling the pathogenic B. anthracis during traditional bacteriological techniques. For 
this, after screening the spores from soil samples, the bacteria were directly used for an optimized
multiplex-PCR. Thus, it is named as a two-stepmethod.

Table 1. Oligonucleotide sequences used in the multiplex-PCR

\begin{tabular}{|c|c|c|c|c|}
\hline Name & Sequence $\left(5^{\prime}-3^{\prime}\right)$ & Target gene & Expected size & Reference \\
\hline Ba-SF & TTCGTCCTGTTATTGCAG & \multirow{3}{*}{$\begin{array}{l}\text { rpoB of } \\
\text { genomic DNA }\end{array}$} & \multirow[t]{3}{*}{ 359-bp and 208-bp } & \multirow{3}{*}{$\begin{array}{l}\text { Ko et al. } \\
\text { (2003) }\end{array}$} \\
\hline BA-RF & GACGATCATYTWGGAAACCG & & & \\
\hline BA-RR & GGNGTYTCRATYGGACACAT & & & \\
\hline Pag-F & GAAGAGTGAGGGTGGATACA & \multirow[t]{2}{*}{ pag of pX01 } & \multirow[t]{2}{*}{ 164-bp } & \multirow[t]{2}{*}{ This study } \\
\hline Pag-R & CATATCCGGTTTAGTCGTTT & & & \\
\hline Cap-F & GTACTTAGAACTTTGTGGTATG & \multirow[t]{2}{*}{ cap of pX02 } & \multirow[t]{2}{*}{ 287-bp } & \multirow[t]{2}{*}{ This study } \\
\hline Cap-R & TTTCGTCTCATTCTACCTCAC & & & \\
\hline
\end{tabular}

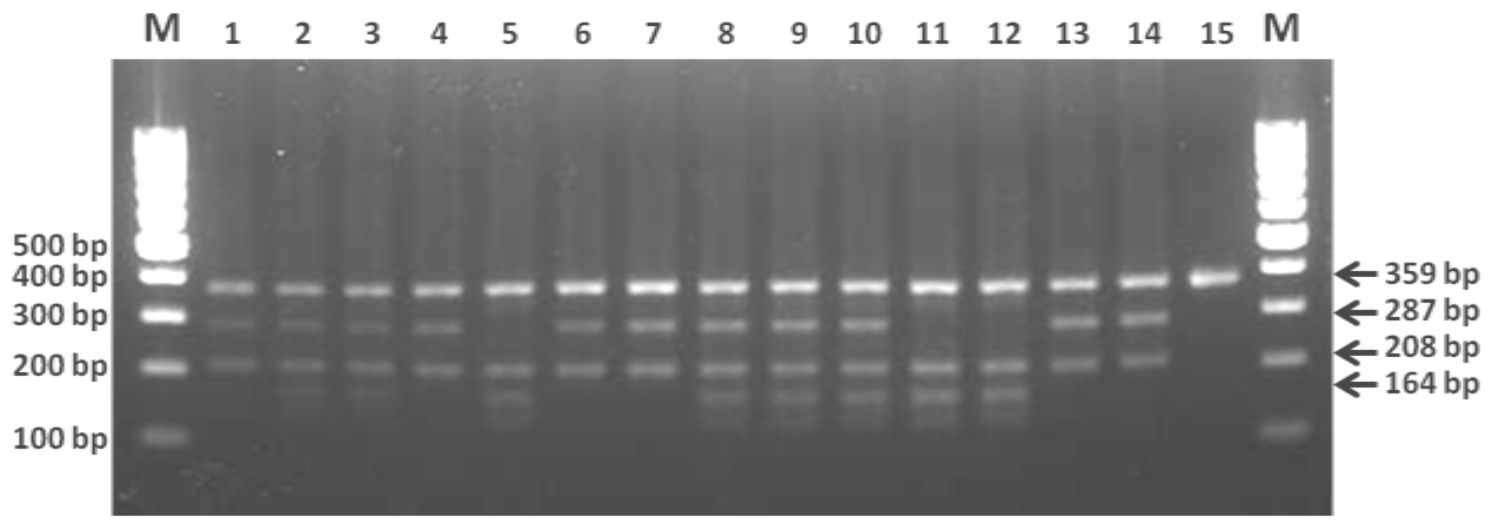

Figure 1. Agarose gel electrophoresis of multiplex-PCR products of $B$. anthracis isolates. $M=100$-bp DNA ladder; Lanes 1, 4, 6, 7, 13 and 14 indicate the isolates as $B$. anthracis containing pX02 plasmid. Lanes - 2, 3, 8, 9 and 10 indicate B. anthracis containing both pX01 and pX02 plasmids. Lanes- 5, 11 and 12 indicated the bacteria as B. anthracis harboring pX01 plasmid. Lane-15 is the negative control having no plasmid but confirmed to be the genus Bacillus.

The multiplex-PCR method targeting rpoB gene of $B$. anthracis genome has been assessed to distinguish the bacteria from a number of related strains including $B$. cereus and $B$. thuringiensis which are considered as the closest neighbors in phylogenetic analysis (Ko et al., 2003). The same primer pairs (Table 1) were used in our experiment. In addition, we designed and used the primer-sets specific for pX01 and pX02 plasmids indicating that our strategy expanded the previous method. In our study, $100 \%$ isolates grown on PLET agar were confirmed as $B$. anthracis (Figure 1). However, variation was found in the presence of toxin-encoding plasmids (Vahedi et al., 2009). Only 5 (6.94\%) isolates contained both pX01 and pX02 plasmids. Six isolates were found to be positive for only pX02 and three were positive for only pX01. On overall evaluation, 8 (57.14\%) harbored pX01 plasmid, and the capsule encoding pX02 plasmid was identified in 11 (78.57\%) isolates (Figure 1). For final validation of our newly developed two-step-method, all the isolates were reidentified as expected by traditional bacteriological and biochemical methods.

\section{Conclusion}

The newly established two-step-method was found sensitive to identify virulent strains of $B$. anthracis. The findings of this study can be used as an easy, 
precise and rapid screening of $B$. anthracis from soil and other environmental samples.

\section{Acknowledgement}

This study was financially supported by Bangladesh Agricultural Research Council (BARC), Dhaka, Bangladesh under Core Research Projects 2011-12 (No. 24; to Nazir, KHMNH).

\section{References}

Ahmed N, Sultana Y, Fatema DSM, Ara K, Begum N, Mostanzid SM, Jubayer S (2010). Anthrax: An emerging zoonotic disease in Bangladesh. Bangladesh J. Med. Microbiol. 4:46-50.

Ahsan MM, Khan MFR, Rahman MB, Hassan J, Chowdhury SMZH, Parvej MS, Jahan M, Nazir KHMNH (2013). Investigation of Bacillus anthracis spore in soil and analysis of environmental parameters related to repeated anthrax outbreak in Sirajganj, Bangladesh. Thai J. Vet. Med. 43:411-416.

Biswas PK, Islam MJ, Shil SK, Chakraborty RK, Ahmed SSU, Christensen JP (2011). Risk factors associated with anthrax in cattle on smallholdings. Epidemiol. Infect. 140:18881895.

Dragon DC, Rennie RP (2001). Evaluation of spore extraction and purification methods for selective recovery of viable Bacillus anthracis spores. Lett. Appl. Microbiol. 33:100-105.

Fasanella A, Garofolo G, Hossain MJ, Shamsuddin M, Blackburn JK, Hugh-Jones M (2013). Bangladesh anthrax outbreaks are probably caused by contaminated livestock feed. Epidemiol. Infect. 141:1021-1028.
Hassan J, Ahsan MM, Rahman MB, Chowdhury SMZH, Parvej MS, Nazir KHMNH (2015a). Factors associated with repeated outbreak of anthrax in Bangladesh: qualitative and quantitative study. J. Adv. Vet. Anim. Res. 2:158-164.

Hassan J, Rahman MB, Chowdhury SMZH, Rabidas SK, Parvej MS, Nazir KHMNH (2015b). ELISA based anthrax antibody titer in cattle induced by locally prepared anthrax vaccine originated from Sterne F-24 strain in Bangladesh. Microbes Health. 4:36-38.

Jula GM, Jabbari A, Vahedi-darmian F (2007). Determination of anthrax foci through isolation of Bacillus anthracis form soil samples of different regions of Iran. Arch. Razi Institute, 62:23-30.

Ko KS, Kim JM, Kim JW, Jung BY, Kim W, Kim IJ, Kook YH (2003). Identification of Bacillus anthracis by $r p o \mathrm{~B}$ sequence analysis and multiplex PCR. J. Clin. Microbiol. 41:29082914.

Kumar S, Tuteja U, Sarika K, Kumar O (2012). A multiplex PCR assay for the simultaneous identification of virulent \& avirulent Bacillus anthracis targeting genes of plasmids \& chromosomal DNA. Indian J. Med. Res. 135:917-919.

Park SH, Oh HB, Seong WK, Kim CW, Cho SY, Yoo CK (2007). Differential analysis of Bacillus anthracis after pX01 plasmid curing and comprehensive data on Bacillus anthracis infection in macrophages and glial cells. Proteomics, 7:3743-3758.

Vahedi F, Jula GM, Kianizadeh M, Mahmoudi M (2009). Characterization of Bacillus anthracis spores isolates from soil by biochemical and multiplex PCR analysis. Eastern Med. Health J. 15:149-156. 